\title{
CHARACTERIZATIONS OF ROTUNDITY AND SMOOTHNESS BY APPROXIMATE ORTHOGONALITIES
}

\author{
Tomasz StypuŁa, Pawę Wójcik
}

\begin{abstract}
In this paper we consider the approximate orthogonalities in real normed spaces. Using the notion of approximate orthogonalities in real normed spaces, we provide some new characterizations of rotundity and smoothness of dual spaces.
\end{abstract}

\section{Introduction}

Let $(X,\|\cdot\|)$ be a real normed space. A well-known theorem states that if $X^{*}$ is rotund, then $X$ is smooth. The aim of this paper is to extend this theorem and to bring some results concerning the approximate orthogonalities.

We define two mappings $\rho_{+}^{\prime}, \rho_{-}^{\prime}: X \times X \rightarrow \mathbb{R}$ :

$$
\rho_{ \pm}^{\prime}(x, y):=\lim _{t \rightarrow 0^{ \pm}} \frac{\|x+t y\|^{2}-\|x\|^{2}}{2 t}=\|x\| \lim _{t \rightarrow 0^{ \pm}} \frac{\|x+t y\|-\|x\|}{t} .
$$

This mappings are called norm derivatives. Convexity of the norm yields that the above definitions are meaningful. Now, we recall their useful properties

Received: 1.03.2016. Revised: 25.05.2016. Accepted: 08.06.2016.

(2010) Mathematics Subject Classification: 46B20, 46C50, 46B10.

Key words and phrases: approximate orthogonality, rotundity, smoothness, dual space, norm derivatives. 
(the proofs can be found in [1] and [4]):

$$
\begin{array}{ll}
\forall_{x, y \in X} \forall_{\alpha \in \mathbb{R}}: & \rho_{ \pm}^{\prime}(x, \alpha x+y)=\alpha\|x\|^{2}+\rho_{ \pm}^{\prime}(x, y) ; \\
\forall_{x, y \in X} \forall_{\alpha \geq 0}: \quad \rho_{ \pm}^{\prime}(\alpha x, y)=\alpha \rho_{ \pm}^{\prime}(x, y)=\rho_{ \pm}^{\prime}(x, \alpha y) ; \\
\forall_{x, y \in X} \forall_{\alpha<0}: \quad \rho_{ \pm}^{\prime}(\alpha x, y)=\alpha \rho_{\mp}^{\prime}(x, y)=\rho_{ \pm}^{\prime}(x, \alpha y) ; \\
\forall_{x, y \in X}: \quad\left|\rho_{ \pm}^{\prime}(x, y)\right| \leq\|x\| \cdot\|y\| ; \\
\forall_{x \in X}: \quad \rho_{ \pm}^{\prime}(x, x)=\|x\|^{2} .
\end{array}
$$

Moreover, mappings $\rho_{+}^{\prime}, \rho_{-}^{\prime}$ are continuous with respect to the second variable, but not necessarily with respect to the first one.

Due to G. Lumer [7] and J.R. Giles [6] in every real normed space $(X,\|\cdot\|)$, there exists a mapping $[\cdot \cdot \cdot]: X \times X \rightarrow \mathbb{R}$ satisfying the following properties:

$$
\begin{aligned}
& \forall_{x, y, z \in X} \forall_{\alpha, \beta \in \mathbb{R}}: \quad[\alpha x+\beta y \mid z]=\alpha[x \mid z]+\beta[y \mid z] ; \\
& \forall_{x, y \in X} \forall_{\alpha \in \mathbb{R}}: \quad[x \mid \alpha y]=\alpha[x \mid y] ; \\
& \forall_{x, y \in X}: \quad|[x \mid y]| \leq\|x\| \cdot\|y\| ; \\
& \forall_{x \in X}: \quad[x \mid x]=\|x\|^{2} .
\end{aligned}
$$

Such a mapping is called a semi-inner product (s.i.p.) in $X$ (generating the norm $\|\cdot\|)$. There may exist infinitely many different semi-inner products in $X$. There is a unique one if and only if $X$ is smooth (i.e., there is a unique supporting hyperplane at each point of the unit sphere $S_{X}$ ). If $X$ is an inner product space, the only s.i.p. on $X$ is the inner product itself.

If $[\cdot \mid \cdot]$ is a given semi inner product in $X$, then

$$
\rho_{-}^{\prime}(x, y) \leq[y \mid x] \leq \rho_{+}^{\prime}(x, y), \quad x, y \in X .
$$

It is known that $X$ is smooth if and only if $\rho_{+}^{\prime}(x, y)=\rho_{-}^{\prime}(x, y)=[y \mid x]$ for all $x, y \in X$.

Let $X^{*}$ denote the set of all linear and continuous functions from $X$ into the field $\mathbb{R}$. Suppose that $x$ is a point on the unit sphere $S_{X}$ and fix the semiinner product $[\cdot \mid \cdot]$. Then the mapping $\varphi_{x}(\cdot):=[\cdot \mid x]$ is a supporting functional at the point $x$. Conversely, every supporting functional at the point $x \in S_{X}$ can be written in this form (cf. [4]).

Applying (1.1) we obtain

$$
\rho_{-}^{\prime}(x, \cdot) \leq \varphi_{x}(\cdot) \leq \rho_{+}^{\prime}(x, \cdot) \text { for all supporting functional } \varphi_{x} \text { at } x .
$$


There are also connections between norm derivatives and the semi-inner products. Namely, the following result holds (cf. [4]).

THeOREm 1.1. Let $(X,\|\cdot\|)$ be a real normed space and $\mathcal{S}_{p}$ the set of all s.i.p. on $X$ which generate the norm $\|\cdot\|$. Then

$$
\begin{aligned}
\rho_{+}^{\prime}(x, y) & =\sup \left\{\varphi_{x}(y): \varphi_{x} \text { is a supporting functional }\right\} \\
& =\sup \left\{\left[y \mid \frac{x}{\|x\|}\right]:[\cdot \mid \cdot] \in \mathcal{S}_{p}\right\}, \\
\rho_{-}^{\prime}(x, y) & =\inf \left\{\varphi_{x}(y): \varphi_{x} \text { is a supporting functional }\right\} \\
& =\inf \left\{\left[y \mid \frac{x}{\|x\|}\right]:[\cdot \mid \cdot] \in \mathcal{S}_{p}\right\},
\end{aligned}
$$

for all vectors $x, y$ in $X$.

Assume that $m \in S_{X}$. Let us consider the set

$$
\mathcal{F}_{m}:=\left\{\varphi \in X^{*}: \varphi \text { is a supporting functional at the point } m\right\} .
$$

Using 1.2, we have

$$
\forall_{u^{*}, w^{*} \in \mathcal{F}_{m}} \forall_{y \in X} \quad\left|u^{*}(y)-w^{*}(y)\right| \leq \rho_{+}^{\prime}(m, y)-\rho_{-}^{\prime}(m, y) .
$$

In a normed space with a given semi inner product $[\cdot \cdot \cdot]$ a semi-orthogonality and an approximate semi-orthogonality ( $\varepsilon$-semi-orthogonality, with $\varepsilon \in[0,1)$ ) of vectors $x$ and $y$ is naturally defined by:

$$
x \perp_{\mathrm{s}} y: \Leftrightarrow[y \mid x]=0 ; \quad \quad x \perp_{\mathrm{s}}^{\varepsilon} y: \Leftrightarrow|[y \mid x]| \leq \varepsilon\|x\| \cdot\|y\| .
$$

The notions of a $\rho_{+}, \rho_{-}$-orthogonality and an approximate $\rho_{+}, \rho_{-}$-orthogonality (with $\varepsilon \in[0,1)$ ) were defined in [2, 3] as follows:

$$
\begin{aligned}
& \perp_{\rho_{+}} y: \Leftrightarrow \quad \rho_{+}^{\prime}(x, y)=0, \quad x \perp_{\rho_{+}}^{\varepsilon} y: \Leftrightarrow \quad\left|\rho_{+}^{\prime}(x, y)\right| \leq \varepsilon\|x\| \cdot\|y\|, \\
& x \perp_{\rho_{-}} y \Leftrightarrow \quad \rho_{-}^{\prime}(x, y)=0, \quad x \perp_{\rho_{-}}^{\varepsilon} y: \Leftrightarrow \quad\left|\rho_{-}^{\prime}(x, y)\right| \leq \varepsilon\|x\| \cdot\|y\| .
\end{aligned}
$$

If $(X,\langle\cdot \mid \cdot\rangle)$ is a real inner product space, then $\langle y \mid x\rangle=[y \mid x]=\rho_{ \pm}^{\prime}(x, y)$ for arbitrary $x, y \in X$. Hence we have $\perp=\perp_{\mathrm{s}}=\perp_{\rho_{+}}=\perp_{\rho_{-}}$and $\perp^{\varepsilon}=\perp_{\mathrm{s}}^{\varepsilon}=$ $\perp_{\rho_{+}}^{\varepsilon}=\perp_{\rho_{-}}^{\varepsilon}$.

We may consider the condition $\forall x, y \in X \quad x \perp_{a}^{\varepsilon} y \Rightarrow x \perp_{b}^{\varepsilon} y$ and to shorten the notation we write $\perp_{a}^{\varepsilon} \subset \perp_{b}^{\varepsilon}$ (for $\perp_{a}^{\varepsilon}, \perp_{b}^{\varepsilon} \in\left\{\perp_{\rho_{-}}^{\varepsilon}, \perp_{\rho_{+}}^{\varepsilon}, \perp_{\mathrm{s}}^{\varepsilon}\right\}$ ). 
Theorem 1.2. Let $X$ be a real normed space and let $\varepsilon \in[0,1)$. Then the following conditions are equivalent
(a) $\perp_{\rho_{+}}^{\varepsilon} \subset \perp_{\rho_{-}}^{\varepsilon}$
(b) $\perp_{\rho_{+}}^{\varepsilon} \supset \perp_{\rho_{-}}^{\varepsilon}$
(c) $\perp_{\rho_{+}}^{\varepsilon}=\perp_{\rho_{-}}^{\varepsilon}$
(j) $\rho_{+}^{\prime}=\rho_{-}^{\prime}$
(d) $\perp_{\rho_{+}}^{\varepsilon_{+}} \subset \perp_{\mathrm{s}}^{\varepsilon}$
(e) $\perp_{\rho_{+}}^{\varepsilon_{+}} \supset \perp_{\mathrm{s}}^{\varepsilon}$
(f) $\perp_{\rho_{+}}^{\varepsilon_{+}}=\perp_{\mathrm{s}}^{\varepsilon}$
(g) $\perp_{\rho_{-}}^{\varepsilon_{+}} \subset \perp_{\mathrm{s}}^{\varepsilon}$
(h) $\perp_{\rho_{-}}^{\varepsilon} \supset \perp_{\mathrm{s}}^{\varepsilon}$
(i) $\perp_{\rho_{-}}^{\varepsilon}=\perp_{\mathrm{s}}^{\varepsilon}$
(k) $X$ is smooth.

The proof of this result was given in [2] in the case of $\varepsilon=0$. In [3, Theorem 3.3] Chmieliński and the second author proved that (a),(b),(c) and (j),(k) are mutually equivalent. The lacking links $(\mathrm{d}) \Leftrightarrow(\mathrm{e}) \Leftrightarrow \ldots \Leftrightarrow(\mathrm{i}) \Leftrightarrow(\mathrm{k})$ were given in [8, Theorem 1]. By Theorem 1.2, the condition $\perp_{\rho_{+}} \subset \perp_{\rho_{-}}\left(\right.$or $\perp_{\rho_{+}}^{\varepsilon} \subset \perp_{\rho_{-}}^{\varepsilon}$ ) yields the smoothness of the norm. The natural question is: What about real normed linear spaces which satisfy $\perp_{\rho_{+}} \subset \perp_{\rho_{-}}^{\varepsilon}$ or $\perp_{\rho_{-}} \subset \perp_{\rho_{+}}^{\varepsilon}$ ? We explore such questions in the next section. Namely, we will prove that the length of the segments on the unit sphere $S_{X^{*}}$ affects the property $\perp_{\rho_{+}} \subset \perp_{\rho_{-}}^{\varepsilon}$ in $X$.

\section{Main results}

Assume that $X$ is a real normed space such that $\operatorname{dim} X \geq 2$. The following conditions are equivalent:
(i) $\perp_{\rho_{+}} \subset \perp_{\rho_{-}}^{\varepsilon}$,
(ii) $\perp_{\rho_{-}} \subset \perp_{\rho_{+}}^{\varepsilon}$.

Indeed, suppose that $\perp_{\rho_{+}} \subset \perp_{\rho_{-}}^{\varepsilon}$ holds and let $x \perp_{\rho_{-}} y$. Applying nd2' we get $-x \perp_{\rho_{+}} y$. Hence $-x \perp_{\rho_{-}}^{\varepsilon} y$. Applying again nd2' we get $x \perp_{\rho_{+}}^{\varepsilon} y$. The proof of the reverse is the same.

Let $S_{X}, S_{X^{*}}$ denote unit spheres in $X, X^{*}$, respectively. We introduce a geometric constant $\mathcal{E}^{\rho}(X)$,

$$
\mathcal{E}^{\rho}(X):=\inf \left\{\varepsilon \in[0,1]: \perp_{\rho_{+}} \subset \perp_{\rho_{-}}^{\varepsilon}\right\} .
$$

Using nd2 we may rewrite the above as

$$
\mathcal{E}^{\rho}(X)=\inf \left\{\varepsilon \in[0,1]: \forall_{x, y \in S_{X}}\left(\rho_{+}^{\prime}(x, y)=0 \Rightarrow\left|\rho_{-}^{\prime}(x, y)\right| \leq \varepsilon\right)\right\} .
$$

Hence

$$
\mathcal{E}^{\rho}(X)=\sup \left\{\left|\rho_{-}^{\prime}(x, y)\right|: x, y \in S_{X}, \rho_{+}^{\prime}(x, y)=0\right\}
$$


and also

$$
\begin{aligned}
\mathcal{E}^{\rho}(X) & =\inf \left\{\varepsilon \in[0,1]: \perp_{\rho_{-}} \subset \perp_{\rho_{+}}^{\varepsilon}\right\} \\
& =\sup \left\{\left|\rho_{+}^{\prime}(x, y)\right|: x, y \in S_{X}, \rho_{-}^{\prime}(x, y)=0\right\}
\end{aligned}
$$

A normed space $(X,\|\cdot\|)$ is rotund or strictly convex if

$$
\forall_{a, b \in S_{X}} \operatorname{conv}\{a, b\} \subset S_{X} \Rightarrow a=b .
$$

We consider a second constant $\mathcal{R}(X)$ defined as follows

$$
\mathcal{R}(X):=\sup \left\{\|a-b\|: \operatorname{conv}\{a, b\} \subset S_{X}\right\} .
$$

We have, of course, $0 \leq \mathcal{E}^{\rho}(X) \leq 1$ and $0 \leq \mathcal{R}(X) \leq 2$. In particular spaces the upper bounds of $\mathcal{E}^{\rho}(X)$ and $\mathcal{R}(X)$ are attained (see Example 2.5). On the other hand, these constants can be arbitrary small (see Example 2.7). Observe that,

$$
X \text { is smooth } \Leftrightarrow \mathcal{E}^{\rho}(X)=0 ; \quad X \text { is rotund } \Leftrightarrow \mathcal{R}(X)=0 .
$$

The first statement follows from Theorem 1.2, and the second is obvious.

It is known (cf. [5]) that for any normed space $X$, if $X^{*}$ is rotund, then $X$ is smooth, which can be written as

$$
\mathcal{R}\left(X^{*}\right)=0 \Rightarrow \mathcal{E}^{\rho}(X)=0
$$

which is a motivation for our results.

Lemma 2.1. Let $\varphi_{x}, \psi_{x}$ be supporting functionals at a point $x \in S_{X}$. Then $\operatorname{conv}\left\{\varphi_{x}, \psi_{x}\right\} \subset \mathcal{F}_{x}$.

Proof. Fix $\lambda \in[0,1]$. It is easy to verify that $\left\|\lambda \varphi_{x}+(1-\lambda) \psi_{x}\right\| \leq 1$. On the other hand we have $\lambda \varphi_{x}(x)+(1-\lambda) \psi_{x}(x)=1$, thus $\left\|\lambda \varphi_{x}+(1-\lambda) \bar{\psi}_{x}\right\|=1$ and hence $\lambda \varphi_{x}+(1-\lambda) \psi_{x} \in \mathcal{F}_{x}$.

Lemma 2.2. Assume that $X$ is reflexive. Let $a^{*}, b^{*} \in S_{X^{*}}$. Suppose that $\operatorname{conv}\left\{a^{*}, b^{*}\right\} \subset S_{X^{*}}$. Then, there is $m \in S_{X}$ such that $\operatorname{conv}\left\{a^{*}, b^{*}\right\} \subset \mathcal{F}_{m}$. 
Proof. By the assumptions, $\left\|a^{*}\right\|=\left\|b^{*}\right\|=1$ and $\left\|\frac{1}{2} a^{*}+\frac{1}{2} b^{*}\right\|=1$. Since $X$ is reflexive, the functional $\frac{1}{2} a^{*}+\frac{1}{2} b^{*}$ attains its norm. Therefore, $\frac{1}{2} a^{*}(m)+\frac{1}{2} b^{*}(m)=1$ for some $m \in S_{X}$. Suppose that $a^{*}(m)<1$. This implies that $b^{*}(m)>1$ which is a contradiction to $\left\|b^{*}\right\|=1$. Therefore, $a^{*}(m)=b^{*}(m)=1$, and consequently $a^{*}, b^{*} \in \mathcal{F}_{m}$. Applying Lemma 2.1 we get $\operatorname{conv}\left\{a^{*}, b^{*}\right\} \subset \mathcal{F}_{m}$.

Now, we are ready to present a generalization of the statement (2.1). The main results of this section are given in the two following theorems.

TheOREM 2.3. For an arbitrary real normed space $X$ we have

$$
\mathcal{E}^{\rho}(X) \leq \mathcal{R}\left(X^{*}\right)
$$

Proof. We have to show that

$$
\mathcal{E}^{\rho}(X) \leq \sup \left\{\left\|u^{*}-w^{*}\right\|: \operatorname{conv}\left\{u^{*}, w^{*}\right\} \subset S_{X^{*}}\right\} .
$$

Assume, contrary to our claim, that

$$
\left\|u^{*}-w^{*}\right\| \leq \varepsilon_{o}<\mathcal{E}^{\rho}(X) \text { for all segments } \operatorname{conv}\left\{u^{*}, w^{*}\right\} \subset S_{X^{*}} .
$$

Suppose that $x \in S_{X}$. Let $\varphi_{x}$ and $\psi_{x}$ be two supporting functionals at the point $x$. Using Lemma 2.1 we get $\operatorname{conv}\left\{\varphi_{x}, \psi_{x}\right\} \subset S_{X^{*}}$. It follows from (2.3) that

$$
\forall_{y \in S_{X}}\left|\varphi_{x}(y)-\psi_{x}(y)\right| \leq \varepsilon_{o} .
$$

Now, fix $y \in S_{X}$. Passing to the supremum over $\varphi_{x} \in \mathcal{F}_{x}$ and passing to the infimum over $\psi_{x} \in \mathcal{F}_{x}$ (see 1.2 and Theorem 1.1), we can obtain $\left|\rho_{+}^{\prime}(x, y)-\rho_{-}^{\prime}(x, y)\right| \leq \varepsilon_{o}$ (for all $x, y \in S_{X}$ ). Now, for arbitrary $x, y \in X$, putting $\frac{x}{\|x\|}, \frac{y}{\|y\|}$ in place of $x, y$ in the above inequality and applying nd2), we get

$$
\forall_{x, y \in X} \quad\left|\rho_{+}^{\prime}(x, y)-\rho_{-}^{\prime}(x, y)\right| \leq \varepsilon_{o}\|x\| \cdot\|y\| .
$$

It follows from 2.5 that $\perp_{\rho_{+}} \subset \perp_{\rho_{-}}^{\varepsilon_{o}}$. Thus we have $\mathcal{E}^{\rho}(X) \leq \varepsilon_{o}$, a contradiction to $\varepsilon_{o}<\mathcal{E}^{\rho}(X)$. The proof is complete.

The inequality 2.2 can be sharp, we will see it in Example 2.5 . 
TheOrem 2.4. Let $X$ be a reflexive Banach space. Then

$$
\mathcal{R}\left(X^{*}\right) \leq 2 \mathcal{E}^{\rho}(X) .
$$

Proof. Define $E_{+}:=\left\{\varepsilon \in[0,1]: \perp_{\rho_{+}} \subset \perp_{\rho_{-}}^{\varepsilon}\right\}$ and fix $\varepsilon \in E_{+}$. Fix $x, y \in X \backslash\{0\}$. Notice that, due to nd1, we have $x \perp_{\rho_{+}}\left(-\frac{\rho_{+}^{\prime}(x, y)}{\|x\|^{2}} x+y\right)$. Therefore $x \perp_{\rho_{-}}^{\varepsilon}\left(-\frac{\rho_{+}^{\prime}(x, y)}{\|x\|^{2}} x+y\right)$, and hence

$$
\begin{aligned}
\left|\rho_{-}^{\prime}\left(x,-\frac{\rho_{+}^{\prime}(x, y)}{\|x\|^{2}} x+y\right)\right| & \leq \varepsilon\|x\| \cdot\left\|-\frac{\rho_{+}^{\prime}(x, y)}{\|x\|^{2}} x+y\right\| \\
& \leq \varepsilon\|x\| \cdot\left(\frac{\left|\rho_{+}^{\prime}(x, y)\right|}{\|x\|^{2}}\|x\|+\|y\|\right) \\
& =\varepsilon\left|\rho_{+}^{\prime}(x, y)\right|+\varepsilon\|x\| \cdot\|y\| \\
& \leq \varepsilon\|x\| \cdot\|y\|+\varepsilon\|x\| \cdot\|y\|=2 \varepsilon\|x\| \cdot\|y\|,
\end{aligned}
$$

by nd3). Thus we obtain

$$
\forall_{x, y \in X} \quad\left|\rho_{+}^{\prime}(x, y)-\rho_{-}^{\prime}(x, y)\right| \leq 2 \varepsilon\|x\| \cdot\|y\| .
$$

Now, we show that $\left\|a^{*}-b^{*}\right\| \leq 2 \varepsilon$ for all segments $\operatorname{conv}\left\{a^{*}, b^{*}\right\} \subset S_{X^{*}}$. Fix a segment $\operatorname{conv}\left\{a^{*}, b^{*}\right\} \subset S_{X^{*}}$. Applying Lemma 2.2 there is a vector $m \in S_{X}$ such that $a^{*}, b^{*} \in \mathcal{F}_{m}$. Thus, we get

$$
\forall y \in S_{X} \quad\left|a^{*}(y)-b^{*}(y)\right| \leq\left|\rho_{+}^{\prime}(m, y)-\rho_{-}^{\prime}(m, y)\right| \leq 2 \varepsilon,
$$

where the first inequality follows from $(1.3)$ and the second from 2.6 . Passing to the supremum over $y \in S_{X}$ we get $\left\|a^{*}-b^{*}\right\| \leq 2 \varepsilon$. Finally, passing to infimum over $\varepsilon \in E_{+}$we get $\left\|a^{*}-b^{*}\right\| \leq 2 \mathcal{E}^{\rho}(X)$ and the result follows.

From Theorem 2.4 we get a sufficient condition for $X$ to be a nonreflexive Banach space. Namely, if $\mathcal{R}\left(X^{*}\right)>2 \mathcal{E}^{\rho}(X)$, then $X$ is not reflexive. In the next example we show that the estimate in Theorem 2.4 is sharp.

Example 2.5. Consider the space $V:=l_{2}^{\infty}=\left(\mathbb{R}^{2},\|\cdot\|_{\infty}\right)$ with the norm $\left\|\left(x_{1}, x_{2}\right)\right\|_{\infty}:=\max \left\{\left|x_{1}\right|,\left|x_{2}\right|\right\}$. Let $(1,1),(-1,0) \in V$. It is easy to check that

$$
(1,1) \perp_{\rho_{+}}(-1,0) \quad \text { and } \quad \rho_{-}^{\prime}((1,1),(-1,0))=-1 .
$$

In particular, this shows that $\perp_{\rho_{+}} \nsubseteq \perp_{\rho_{-}}^{\varepsilon}$ for all $\varepsilon \in[0,1)$. Therefore $\mathcal{E}^{\rho}(V)=$ 1. On the other hand, we have $V^{*}=\left(l_{2}^{\infty}\right)^{*}=l_{2}^{1}$ where $l_{2}^{1}=\left(\mathbb{R}^{2},\|\cdot\|_{1}\right)$ with 
the norm $\left\|\left(x_{1}, x_{2}\right)\right\|_{1}:=\left|x_{1}\right|+\left|x_{2}\right|$. For the vectors $(1,0),(0,1) \in V^{*}$ we have $\operatorname{conv}\{(1,0),(0,1)\} \subset S_{V^{*}}$ and $\|(1,0)-(0,1)\|=2$, thus $\mathcal{R}\left(V^{*}\right)=2$. Therefore $\mathcal{R}\left(V^{*}\right)=2 \mathcal{E}^{\rho}(V)$.

The reflexivity is necessary to obtain the inequality $\mathcal{R}\left(X^{*}\right) \leq 2 \mathcal{E}^{\rho}(X)$. Indeed, there exists a smooth nonreflexive Banach space $Y$ such that the space $Y^{*}$ is not rotund (see [5]). Thus $\mathcal{R}\left(Y^{*}\right)>0$ and $\mathcal{E}^{\rho}(Y)=0$. Hence $\mathcal{R}\left(Y^{*}\right)>2 \mathcal{E}^{\rho}(Y)$.

Finally, the rotundity of $X^{*}$ can be characterized as follows. Combining Theorems 2.3 and 2.4 we obtain the following corollary.

Corollary 2.6. For an arbitrary reflexive Banach space $X$ we have

$$
\mathcal{E}^{\rho}(X) \leq \mathcal{R}\left(X^{*}\right) \leq 2 \mathcal{E}^{\rho}(X) .
$$

Hence,

(i) if $X$ is reflexive, $X^{*}$ is rotund if and only if $X$ is smooth;

(ii) if $X$ is reflexive, $X^{*}$ is smooth if and only if $X$ is rotund.

Proof. It follows from (2.7) that (i) holds. Using (2.7) for $X^{*}$ we have $\mathcal{E}^{\rho}\left(X^{*}\right) \leq \mathcal{R}(X) \leq 2 \mathcal{E}^{\rho}\left(X^{*}\right)$ and we can easily obtain (ii).

ExAmple 2.7. Fix $\varepsilon \in(0,1)$ and consider the space $Y:=\mathbb{R}^{2}$ with the norm defined by its unit ball

$$
K:=\left\{\left(x_{1}, x_{2}\right) \in \mathbb{R}^{2}: x_{1}^{2}+x_{2}^{2} \leq 1 \wedge\left|x_{1}\right| \leq \sqrt{1-\left(\frac{\varepsilon}{2}\right)^{2}}\right\} .
$$

It is easy to check that $\mathcal{R}(Y)=\varepsilon$. Since $\operatorname{dim} Y<\infty$, then there exists a space $X$ such that $X^{*}=Y$. Thus we have $\mathcal{R}\left(X^{*}\right)=\varepsilon$. According to Corollary 2.6 we have $\frac{\varepsilon}{2} \leq \mathcal{E}^{\rho}(X) \leq \varepsilon$.

\section{References}

[1] Alsina C., Sikorska J., Santos Tomás M., Norm Derivatives and Characterizations of Inner Product Spaces, World Scientific, Hackensack, New Jersay, 2009.

[2] Chmieliński J., Wójcik P., On a $\rho$-orthogonality, Aequationes Math. 80 (2010), 45-55.

[3] Chmieliński J., Wójcik P., $\rho$-orthogonality and its preservation - revisited, in: Recent Developments in Functional Equation and Inequalities, Banach Center Publ., Polish Acad. Sci. Inst. Math., Warsaw, 2013, pp. 17-30. 
[4] Dragomir S.S., Semi-inner products and applications, Nova Science Publishers, Inc., Hauppauge, New York, 2004.

[5] Day M.M., Normed linear spaces, Ergeb. Math. Grenzgeb. 21, Springer, New YorkHeidelberg, 1973.

[6] Giles J.R., Classes of semi-inner-product spaces, Trans. Amer. Math. Soc. 129 (1967), 436-446.

[7] Lumer G., Semi-inner-product spaces, Trans. Amer. Math. Soc. 100 (1961), 29-43.

[8] Wójcik P., Characterizations of smooth spaces by approximate orthogonalities, Aequationes Math. 89 (2015), 1189-1194.

Institute of Mathematics

Pedagogical University of Cracow

PODCHORȦ̧̇̇YH 2

30-084 KRAKÓW

POLAND

e-mail: tomek.stypula@gmail.com

e-mail: pwojcik@up.krakow.pl 